\title{
Pressure-induced magnetic order in FeSe: A muon spin rotation study
}

\author{
Rustem Khasanov, ${ }^{1, *}$ Zurab Guguchia, ${ }^{1}$ Alex Amato, ${ }^{1}$ Elvezio Morenzoni, ${ }^{1}$ Xiaoli Dong, ${ }^{2}$ Fang Zhou, ${ }^{2}$ and Zhongxian Zhao ${ }^{2}$ \\ ${ }^{1}$ Laboratory for Muon Spin Spectroscopy, Paul Scherrer Institut, 5232 Villigen PSI, Switzerland \\ ${ }^{2}$ Beijing National Laboratory for Condensed Matter Physics, Institute of Physics \& University of Chinese Academy of Sciences, \\ CAS, Beijing 100190, China
}

(Received 28 October 2016; published 18 May 2017)

\begin{abstract}
The magnetic order induced by the pressure was studied in FeSe by means of muon spin rotation $(\mu \mathrm{SR})$ technique. By following the evolution of the oscillatory part of the $\mu$ SR signal as a function of angle between the initial muon spin polarization and 101 axis of the studied FeSe sample, it was found that the pressure-induced magnetic order in FeSe corresponds either to the collinear (single-stripe) antiferromagnetic order as observed in parent compounds of various FeAs-based superconductors or to the bi-collinear order as obtained in the FeTe system, but with the Fe spins turned by $45^{\circ}$ within the $a b$ plane. The value of the magnetic moment per Fe atom was estimated to be $\simeq 0.13-0.14 \mu_{\mathrm{B}}$ at $p \simeq 1.9 \mathrm{GPa}$.
\end{abstract}

DOI: 10.1103/PhysRevB.95.180504

Since their discovery in 2008 [1], iron chalcogenide superconductors have attracted much interest. Being composed of a single layer of square Fe lattice tetrahedrally coordinated by chalcogene $(\mathrm{Ch})$ atoms $(\mathrm{Ch}=\mathrm{Se}, \mathrm{Te}, \mathrm{S})$, $\mathrm{FeCh}$ consists just of fundamental building blocks of $\mathrm{Fe}-$ based high-temperature superconductors (Fe-HTS). The iron selenide, FeSe, superconducts at ambient conditions with the transition temperature $T_{c} \simeq 8 \mathrm{~K}$. Early muon spin rotation $(\mu \mathrm{SR})$ experiments on FeSe revealed that the system is nonmagnetic at ambient pressure down to $T \simeq 0.02 \mathrm{~K}$ [2]. The first pressure experiments also do not detect the magnetic order up to pressures at least $p \sim 20 \mathrm{GPa}$ [3]. This is in striking contrast to the other Fe-HTSs that usually exhibit static magnetic order in the parent compound [4,5]. Shortly after, the NMR studies showed a wipeout of the signal that revealed an incipient magnetic phase transition under pressure [6]. It was further realized that pressure promotes the static magnetism in FeSe which was confirmed in $\mu$ SR experiments by Bendele and coworkers $[7,8]$. The static magnetic order which compete with superconductivity was set in above $p \simeq 0.8 \mathrm{GPa}$ and both ground states were found to coexist on an atomic length for pressures exceeding $\sim 1.2 \mathrm{GPa}$.

So far the only confirmation of pressure-induced bulk magnetic order in FeSe was obtained from $\mu$ SR data $[7,8]$. Only very recently the appearance of bulk magnetism in high-quality FeSe single crystalline samples was confirmed in Mössbauer under pressure experiments by Kothapalli et al. [9] and NMR studies of Wang et al. [10]. It should be emphasized, however, that the exact magnetic-spin arrangement in FeSe is still unknown. The problem stems from the low value of the ordered magnetic moment on the Fe site $\left(m_{\mathrm{Fe}}\right)$. Following $\mu \mathrm{SR}$ studies, for pressures $p \lesssim 2.5 \mathrm{GPa} m_{\mathrm{Fe}}$ do not exceed $0.2 \mu_{\mathrm{B}}$ [7]. Recent Mössbauer experiment results in a similar estimate of $m_{\mathrm{Fe}}$ for $p \lesssim 4 \mathrm{GPa}$ [9]. Such small values of $m_{\mathrm{Fe}}$ make the determination of the magnetic structure by means of neutron experiments to be quite challenging. We are only aware of one neutron diffraction measurement allowing us to set the upper limit of $m_{\mathrm{Fe}}<0.5-0.7 \mu_{\mathrm{B}}$ for pressures $p \lesssim 4.5 \mathrm{GPa}$ [7].

*rustem.khasanov@psi.ch
Experimentally, the antiferromagnetic (AFM) collinear structure consistent of stripes of parallel spins (the 'Collinear 1' structure, Fig. 1) was established for parent compounds of FeAs-based Fe-HTSs [5,11]. The so-called 'bi-collinear' order (denoted as 'Bi-collinear1,' Fig. 1) was resolved for parent compounds of iron tellurides $[5,12,13]$. For FeSe the direct measurements are still missing and only theoretical considerations were made until now. For bulk FeSe the 'Collinear2' type of order was proposed in Ref. [14] based on first principle calculations. The AFM 'Pair-checkerboard' order in bulk and monolayer $\mathrm{FeSe}$ was considered by $\mathrm{Cao}$ et al. [15]. For the monolayer and the bi-layer FeSe films the 'Checkerboard' and the mixture of the "Checkerboard" and "Collinear1" orders were predicted in Ref. [16]. The purely 'Pair-checkerboard' order in bulk and thin-film FeSe was obtained in Ref. [17]. The authors of Ref. [18] have reported that the 'Pair-checkerboard' and the 'Collinear1' stripe orders are realized in thin-film and bulk FeSe, respectively.

The aim of this paper is to identify the type of the pressure-induced magnetic order in FeSe by means of muon spin rotation technique. In addition to that mentioned above, the 'Bi-collinear2' order which differs from the 'Bi-collinear 1' one by spins turned to $45^{\circ}$ (see Fig. 1 and Refs. $[16,17]$ ) as well as several magnetic structures suggested by Christensen et al. [19] for various electron- and hole-doped Fe-HTSs were considered. This includes the collinear order denoted as 'Collinear-Z', two types of spin vortex crystal (SVC) phases, and the charge-spin density wave (CSDW) phase. Note that SVC1, SVC2, and CSDW phases preserve the tetragonal symmetry of the system, while the rest of the structures could be observed in both the orthorhombic and the tetragonal magnetic unit cells. By following the evolution of the oscillatory part of the $\mu$ SR signal as a function of angle between the initial muon spin polarization and 101 axis of studied sample [20], it was shown that the pressureinduced magnetic order in FeSe may correspond either to the "Collinear1" or the 'Bi-collinear2' type of the order (see Fig. 1).

The 101-axis oriented FeSe sample growth procedure as well as the details of $\mu \mathrm{SR}$ under pressure experiments are summarized in the Supplemental Material [21] part. Although FeSe crystal used in our study shows the single 101 orientation, 

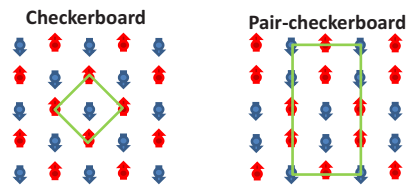

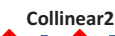

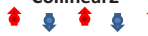

- 15

- 5

$-1$

- 15

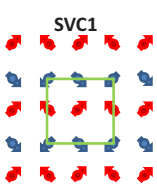

FIG. 1. The in-plane view of ten magnetic phases tested in the present study. The red and the blue arrows (circles) represent $\mathrm{Fe}$ spins. Green rectangles denote the in-plane magnetic unit cell. SVC1, SVC2, and CSDW phases preserve the tetragonal symmetry of the system, while the rest of the structures could be observed in both the orthorhombic and the tetragonal magnetic unit cells.

it is, strictly speaking, very close in shape to a powder with strong preferred orientation. Indeed, no Laue reflection patterns were observed on the studied sample. To the best our knowledge, this is common for FeSe crystals grown by flux or floating-zone methods.

Figure 2(a) shows the schematic of the experiment. The cylindrically shaped FeSe sample, placed inside the pressure cell, was rotated in a way allowing us to change the angle $\theta$ between the initial muon spin polarization $P(0)$ and 101 crystallographic axis of the sample. Typical zero-field muon time spectra measured at $T=15 \mathrm{~K}$ and $p \simeq 1.9 \mathrm{GPa}$ for $\theta=$ $-10^{\circ}$ and $-100^{\circ}$ are shown in Fig. 2. The solid lines are fits
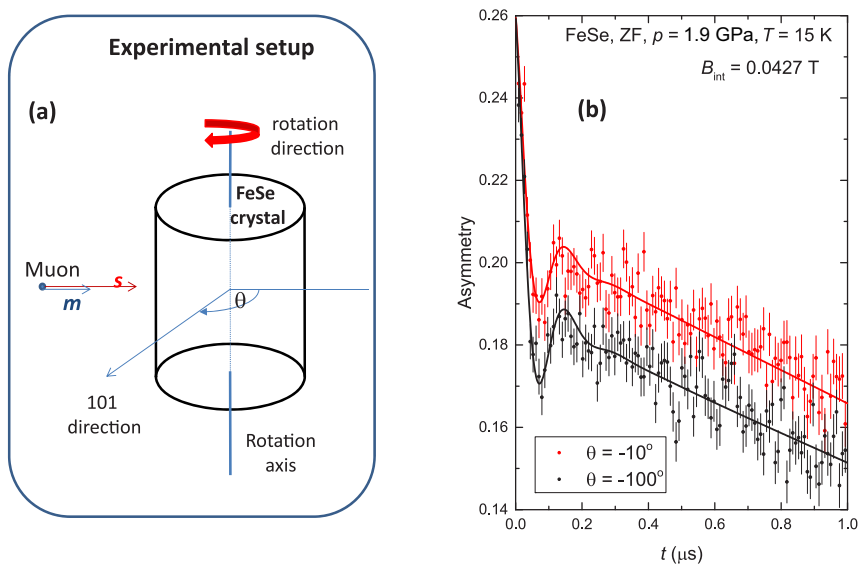

FIG. 2. (a) The schematic of the experiment. The rotation of cylindrically shaped FeSe sample placed inside the pressure cell allows us to change the angle $\theta$ between the initial muon spin polarization and 101 crystallographic axis. The red and blue vectors denote the momentum and the initial spin direction (initial muon-spin polarization) of implanted muons, respectively. (b) ZF- $\mu$ SR time spectra measured at $T=15 \mathrm{~K}, p=1.9 \mathrm{GPa}$ for $\theta=-10^{\circ}$ (red symbols) and $-100^{\circ}$ (black symbols). The solid lines are fits by using Eq. (1). of the following function to the experimental data:

$$
A(t)=A_{s}(0) P_{s}(t)+A_{p c}(0) P_{p c}(t) .
$$

Here $A_{s}(0)$ and $A_{p c}(0)$ are the initial asymmetries and $P_{s}(t)$ and $P_{p c}(t)$ are the muon spin polarizations belonging to the sample and the pressure cell, respectively. The polarization of the pressure cell $P_{p c}(t)$ was obtained in a separated set of experiments [22]. The polarization of the sample was described by the following functional form:

$$
P_{s}(t)=f_{\mathrm{osc}} e^{-\lambda_{T} t} \cos \left(\gamma_{\mu} B_{\mathrm{int}} t\right)+\left(1-f_{\mathrm{osc}}\right) e^{-\lambda_{L} t} .
$$

Here $B_{\text {int }}$ is the internal field on the muon stopping site, $\gamma_{\mu}=2 \pi 135.5 \mathrm{MHz} / \mathrm{T}$ is the muon gyromagnetic ratio, and $\lambda_{T}$ and $\lambda_{L}$ are the transverse and the longitudinal exponential relaxation rates, respectively. The oscillating $\left(f_{\text {osc }}\right)$ and nonoscillating $\left(1-f_{\text {osc }}\right) \mu$ SR signal fractions originate from the magnetic field components which are transversal to the initial muon spin polarization and cause a precession $\left[B_{\text {int }} \perp\right.$ $P(0)]$ and the nonprecessing longitudinal field components $\left[B_{\text {int }} \| P(0)\right]$, respectively. Note that in the powder sample, where all possible angles between $B_{\text {int }}$ and $P(0)$ are equally possible, $f_{\text {osc }} \equiv 2 / 3$. In the single crystalline sample the value of $f_{\text {osc }}$ may vary from 1 , in the $B_{\text {int }} \perp P(0)$ case, to 0 for $B_{\text {int }} \| P(0)$.

From the experimental data presented in Fig. 2(b) two important points emerge: (i) The spontaneous muon spin precession with $B_{\text {int }} \simeq 42.7 \mathrm{mT}$ is clearly detected on the $\mathrm{ZF}$ $\mu \mathrm{SR}$ time spectra. Consequently, the static magnetic order in FeSe studied here is established below the Néel temperature $T_{N}$ in agreement with the results of previous $\mu$ SR experiments on FeSe powders [7,8] and recent Mössbauer experiments of high-quality single crystals [9]. (ii) The value of the oscillatory component $f_{\text {osc }}$ depends on the angle between $P(0)$ and the 101 axis of the sample. $f_{\text {osc }}$ for $\theta=-10^{\circ}$ is obviously smaller than that for $\theta=-100^{\circ}$. Consequently, by measuring $f_{\text {osc }}$ as a function of $\theta$, the direction of the internal field $B_{\text {int }}$ on the muon stopping position might be determined.

The dependence of $f_{\text {osc }}$ on $\theta$ is shown in Fig. 3(a). It has $180^{\circ}$ periodicity with the maximum $\left(f_{\mathrm{osc}}^{\max }\right)$ and minimum
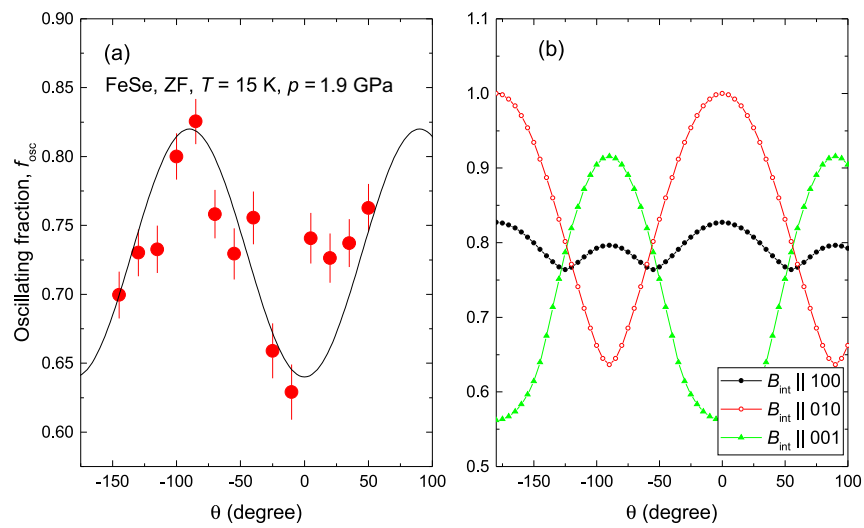

FIG. 3. (a) Angular dependence of the oscillating fraction $f_{\text {osc }}$ in FeSe sample at $T=15 \mathrm{~K}$ and $p=1.9 \mathrm{GPa}$. (b) $f_{\text {osc }}(\theta)$ calculated for the internal field $B_{\text {int }}$ aligned along 100 (black), 010 (red), and 001 (green) crystallographic directions (see the Supplemental Material [21] part for details). 


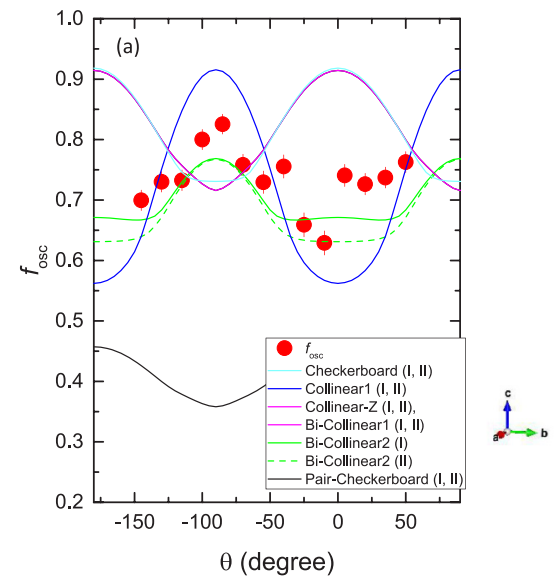

(b)

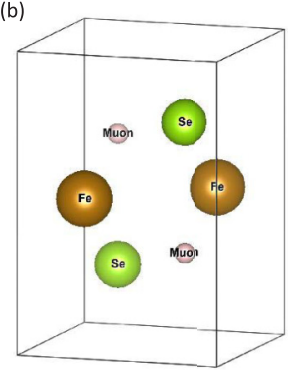

FIG. 4. (a) $f_{\text {osc }}$ as a function of $\theta$ calculated for 'Checkerboard,' 'Collinear1,' 'Bi-collinear1,' 'Bi-collinear2' and 'Collinear-Z' structures. "I" and "II" correspond to the magnetic unit cell to be the same or doubled in comparison to the chemical one along the $c$ axis. (b) The FeSe unit cell (crystallographic group $P 4 / \mathrm{nmm} \mathrm{129}$, origin choice 2) [23]. Muons are on the $2 \mathrm{c}\left[(1 / 4,1 / 4, z), z_{\mu}=0.84\right]$ Wyckoff position. The structure is visualized by using the VESTA package [24].

$\left(f_{\text {osc }}^{\min }\right)$ corresponding to $\theta=90^{\circ}+n \cdot 180^{\circ}$ and $n \cdot 180^{\circ}(n$ is the integer number), respectively. Bendele et al. [7] have considered the collinear (single-stripe) AFM order in FeSe ('Collinear1,' Fig. 1) and shown that in such a case $B_{\text {int }}$ is aligned along the crystallographic $c$ axis. The comparison of $f_{\text {osc }}(\theta)$ with that obtained theoretically for $B_{\text {int }} \| 100$, 010, and 001 crystallographic directions (Fig. 3(b) and the Supplemental Material [21]) is consistent with this statement. Indeed, for $B_{\text {int }} \| 001$ the period, the values of $\theta$ corresponding to the minimum and maximum of $f_{\mathrm{osc}}(\theta)$ are just the same as they observed experimentally. The theoretically calculated $f_{\text {osc }}^{\max }=0.92$ and $f_{\text {osc }}^{\text {min }}=0.57$ are less than $10 \%$ different from the experimentally obtained 0.83 and 0.62 (see Fig. 3). Such a small difference could be explained by some misalignment of the 101 axis between different crystallites. As illustrated in Ref. [20], FeSe samples prepared similarly to the one used in our studies are characterized by a high level of texture which results in a strong broadening of Bragg reflection peaks.

The above obtained consistency between $f_{\text {osc }}(\theta)$ and 'Collinear1' type of order do not allow us, however, to make any firm conclusion about other magnetic phases as they presented in Fig. 1. To obtain more quantitative information, calculations of corresponding internal fields at muon stopping sites were carried out. The muon sites in FeSe were previously calculated by Bendele et al. [7] by using the modified Thomas Fermi approach. There are two equivalent minima in the unit cell corresponding to the $2 \mathrm{c}$ $\left[(1 / 4,1 / 4, z), z_{\mu}=0.84\right.$; according to the crystallographic group $P 4 / \mathrm{nmm} 129$, origin choice 2] Wyckoff position [see Fig. 4(b)].

The spontaneous local field for the muon site $i$ was assumed to be entirely determined by the dipolar component:

$$
\mathbf{B}_{\mathrm{int}, i} \simeq \mathbf{B}_{\mathrm{dip}, i}
$$

with $B_{\text {dip }}$ at position $\mathbf{r}$ within the lattice unit cell calculated via $[25,26]$ :

$$
B_{\mathrm{dip}}^{\alpha}(\mathbf{r})=\frac{\mu_{0}}{4 \pi} \sum_{j, \beta} \frac{m_{j}^{\beta}}{R_{i}^{3}}\left(\frac{3 R_{j}^{\alpha} R_{i}^{\beta}}{R_{j}^{2}}-\delta^{\alpha \beta}\right) .
$$

Here $\mathbf{R}_{j}=\mathbf{r}-\mathbf{r}_{j}, \alpha$ and $\beta$ denote the vector components $x, y$, and $z, \mathbf{r}_{j}$ is the position of $j$ th magnetic ion in the unit cell, and $m_{j}^{\beta}$ is the corresponding magnetic moment. The summation is taken over a sufficiently large Lorentz sphere of radius $R_{L}$. Note that Eq. (3) differs from its general form which includes also the so-called contact field term $\mathbf{B}_{\text {cont }}=A_{\text {cont }} \sum_{k=1}^{N} \mathbf{m}_{k}$ ( $A_{\text {cont }}$ is the contact constant and the summation is made over the $N$ nearest neighboring magnetic moments) $[27,28]$. Thanks to the 2c Wyckoff position of the muon in FeSe lattice [Fig. 4(b)], the sum of $\mathbf{m}_{k}$ becomes zero for all structures presented in Fig. 1 with the magnetic unit cell doubled in comparison with the chemical one along the crystallographic $c$ directions and for all structures except both ' $\mathrm{Bi}$-collinear' ones for a case without doubling.

The results of internal field calculations for various magnetic structures presented in Fig. 1 and $m_{\mathrm{Fe}}=1 \mu_{\mathrm{B}}$ are summarized in Table I. The part of the data denoted as "I" corresponds to the case when the magnetic and the chemical unit cell have similar $c$-axis constants (the magnetic order along the $c$ axis is ferromagnetic). For the part denoted as "II" the magnetic unit cell along the $c$ direction is doubled in comparison to the chemical one and the magnetic order along the $c$ axis becomes antiferromagnetic. The last column shows the value of $m_{\mathrm{Fe}}$ as calculated from the experimentally obtained $B_{\text {int }} \simeq 0.0427$ T [see Fig. 2(b)].

Several magnetic phases could be excluded from the consideration based entirely on the dipolar field calculations. The 'Collinear2' structure results in zero internal field on the muon stopping position. The 'SVC1' and 'SVC2' result in two different $B_{\text {int }}$ 's. Both these findings are inconsistent with the experimentally observed single finite internal field value (see Fig. 2(b) and Refs. [7,8]). Values of $m_{\mathrm{Fe}}$ for 'CSDW' structure were estimated to be 0.70 and $1.04 \mu_{\mathrm{B}}$ for the magnetic unit cell with and without doubling along the crystallographic $c$ direction, respectively. These values are bigger then the upper estimate of $m_{\mathrm{Fe}}<0.5-0.7 \mu_{\mathrm{B}}$ set in neutron diffraction experiment [7].

Following the above discussion, our data are consistent with the internal field on the muon stopping position aligned along the crystallographic $c$ direction. Among the magnetic phases left, the 'Collinear1' phase, which was already proposed in Ref. [7], satisfy such criteria. In order to check if the rest of the phases ('Checkerboard,' 'Pair-checkerboard,' 'Bi-collinear1,' 'Bi-collinear2,' and 'Collinear-Z') could be consistent with the experiment, the corresponding $f_{\text {osc }}(\theta)$ dependencies were calculated [Fig. 4(a)]. Since the AFM order in Fe-HTSs is generally preceded by a tetragonal-to-orthorhombic lattice distortion [5], the twinning effects (rotation of the structure within the $a b$ plane by $90^{\circ}$ ) were also considered. The half of the sample volume was assumed to be 101-axis oriented and another half to be 011-axis oriented. The results presented in Fig. 4 imply that two type of orders, namely the 'Collinear1' and 'Bi-collinear2' become consistent with the experiment. It is worth it to emphasize here that the simulated curves 
TABLE I. Summary of the internal field calculations for FeSe. The in-plane arrangement of Fe spins for each magnetic phase are presented in Fig. 1 in the main text. The cases denoted as "I" and "II" correspond to the magnetic unit cell to be the same or doubled in comparison to the chemical one along the $c$ axis, respectively. Question marks correspond to the unknown field orientation which cancels due to symmetry reasons.

\begin{tabular}{lcccc}
\hline \hline Magnetic phase & $\begin{array}{c}\text { Number of } \\
\text { muon sites }\end{array}$ & $\begin{array}{c}\text { Number of } \\
\text { internal fields }\end{array}$ & $\begin{array}{c}B_{\text {int }} \text { per } 1 \mu_{\mathrm{B}} \\
\text { per Fe atom }\end{array}$ & $\begin{array}{c}\text { Moment per } \\
\text { Fe atom }\end{array}$ \\
\hline Checkerboard (I/II) & $2 / 2$ & $2 / 1$ & $0.454 / 0452 \mathrm{~T}$ & $0.094 / 0.094 \mu_{\mathrm{B}}$ \\
Pair-checkerboard (I/II) & $8 / 8$ & $3 / 3$ & $0.492 ; 0 / 0.486 ; 0 \mathrm{~T}$ & $0.087 ; ? ? / 0.088 ; ? ? \mu_{\mathrm{B}}$ \\
Collinear1 (I/II) & $2 / 2$ & $1 / 2$ & $0.309 / 0.323 \mathrm{~T}$ & $0.138 / 0.132 \mu_{\mathrm{B}}$ \\
Collinear2 (I/II) & $2 / 2$ & $1 / 1$ & $0 / 0 \mathrm{~T}$ & $? ? / ? ?$ \\
Bi-collinear1 (I/II) & $4 / 4$ & $2 / 2$ & $0.324 / 0.323 \mathrm{~T}$ & $0.138 / 0.132 \mu_{\mathrm{B}}$ \\
Bi-collinear2 (I/II) & $4 / 4$ & $2 / 2$ & $0.344 / 0.338 \mathrm{~T}$ & $0.124 / 0.126 \mu_{\mathrm{B}}$ \\
Collinear-Z (I/II) & $2 / 2$ & $2 / 2$ & $0.077 / 0.117 \mathrm{~T}$ & $0.554 / 0.365 \mu_{\mathrm{B}}$ \\
SVC1 (I/II) & $4 / 4$ & $4 / 4$ & $0.032 ; 0.076 / 0.057 ; 0.108 \mathrm{~T}$ & $1.334 ; 0.562 / 0.749 ; 0.395 \mu_{\mathrm{B}}$ \\
SVC2 (I/II) & $4 / 4$ & $4 / 4$ & $0.0019 ; 0.024 / 0.0019 ; 0.024 \mathrm{~T}$ & $22.5 ; 1.78 / 22.5 ; 1.78 \mu_{\mathrm{B}}$ \\
CSDW (I/II) & $4 / 4$ & $4 / 4$ & $0.041 / 0.061 \mathrm{~T}$ & $1.041 / 0.70 \mu_{\mathrm{B}}$ \\
\hline \hline
\end{tabular}

presented in Fig. 4(a) were obtained by assuming the perfect orientation of 101 axis of the sample in accordance with the initial muon spin direction. In reality, as is shown in Ref. [20], the 101 axis misalignment is of the order of 5 degrees in terms of FWHM for x-ray rocking curve. The sample alignment inside the pressure cell could also be not perfectly controlled. Such effects may lead to the reduction of the oscillation amplitude of $f_{\text {osc }}$ as a function of $\theta$.

Based entirely on the experimental data one cannot distinguish between two suggested above magnetic structures. There are few arguments, however, in favor of 'Collinear1' rather than 'Bi-collinear2' type of magnetic order. (i) Strong commensurate spin fluctuations with an in-plane wave vector $\mathbf{q}=(\pi, 0)$ were observed recently in FeSe at ambient pressure $[29,30]$. Note that the in-plane $\mathbf{q}=(\pi, 0)$ corresponds to the stripe-like 'Collinear1' type of magnetic order. (ii) Recent $a b$ initio calculations have attributed the absence of static magnetic order at ambient pressure in FeSe to competition between different magnetic ordering vectors and shown that the application of pressure lifts this near degeneracy, leading to a $(\pi, 0)$ stripe order [31]. (iii) The $(\pi, 0)$ nature of the pressure-induced magnetic state in FeSe is supported by the Fermi surface reconstruction reported in quantum oscillations experiments [32]. (iv) The X-ray diffraction experiments of Kothapalli et al. [9] show that the magnetic order in FeSe breaks the tetragonal symmetry of the lattice in the same manner as the stripe-type magnetic order in the other ironbased materials $[5,11]$.
To conclude, the magnetic order induced by the pressure was studied in the 101 oriented FeSe sample by means of muon spin rotation. By following the evolution of the oscillatory part of the $\mu \mathrm{SR}$ signal as a function of angle between the initial muon spin polarization and the 101 axis of the studied sample, it was found that the pressure-induced magnetic order in FeSe corresponds either to the collinear (single-stripe) antiferromagnetic order as observed in parent compounds of various FeAs-based superconductors or to the bi-collinear order as obtained in the FeTe system, but with the Fe spins turned by $45^{\circ}$ within the $a b$ plane. The value of the magnetic moment per Fe atom was estimated to be $\simeq 0.13-0.14 \mu_{\mathrm{B}}$ at $p \simeq 1.9 \mathrm{GPa}$.

Note added. After submitting the paper we become aware of the accepted version of Ref. [10] where the authors have reported the consistency of their NMR data with the collinear (single-stripe) antiferromagnetic order.

The work was performed at the Swiss Muon Source $(\mathrm{S} \mu \mathrm{S})$, Paul Scherrer Institute (PSI, Switzerland). R.K. acknowledges helpful discussions with Rafael Fernandes. The work of Z.G. was supported by the Swiss National Science Foundation (SNF-Grant No. 200021-149486). The work of X.D., F.Z., and Z.Z. was supported by the "National Key Research and Development Program of China (Grant 2016YFA0300301)," the "Strategic Priority Research Program (B)" of the Chinese Academy of Sciences (Grant No. XDB07020100), and the Natural Science Foundation of China (Grant No. 11574370).
[1] F. C. Hsu, Y. Y. Luo, K. W. Yeh, T. K. Chen, T. W. Huang, P. M. Wu, Z. C. Lee, Y. L. Huang, Y. Y. Chu, D. C. Yan, and M. K. Wu, Proc. Natl. Acad. Sci. USA 105, 14262 (2008).

[2] R. Khasanov, K. Conder, E. Pomjakushina, A. Amato, C. Baines, Z. Bukowski, J. Karpinski, S. Katrych, H.-H. Klauss, H. Luetkens, A. Shengelaya, and N. D. Zhigadlo, Phys. Rev. B 78, 220510(R) (2008).

[3] S. Medvedev, T. M. McQueen, I. A. Troyan T. Palasyuk, M. I. Eremets, R. J. Cava, S. Naghavi, F. Casper, V.
Ksenofontov, G. Wortmann, and C. Felser, Nat. Mater. 8, 630 (2009).

[4] B. Büchner and C. Hess, Nat. Mater. 8, 615 (2009).

[5] P. Dai, Rev. Mod. Phys. 87, 855 (2015).

[6] T. Imai, K. Ahilan, F. L. Ning, T. M. McQueen, and R. J. Cava, Phys. Rev. Lett. 102, 177005 (2009).

[7] M. Bendele, A. Ichsanow, Yu. Pashkevich, L. Keller, Th. Strässle, A. Gusev, E. Pomjakushina, K. Conder, R. Khasanov, and H. Keller, Phys. Rev. B 85, 064517 (2012). 
[8] M. Bendele, A. Amato, K. Conder, M. Elender, H. Keller, H.-H. Klauss, H. Luetkens, E. Pomjakushina, A. Raselli, and R. Khasanov, Phys. Rev. Lett. 104, 087003 (2010).

[9] K. Kothapalli, A. E. Böhmer, W. T. Jayasekara, B. G. Ueland, P. Das, A. Sapkota, V. Taufour, Y. Xiao, E. E. Alp, S. L. Bud'ko, P. C. Canfield, A. Kreyssig, and A. I. Goldman, Nat. Commun. 7, 12728 (2016)

[10] P. S. Wang, S. S. Sun, Y. Cui, W. H. Song, T. R. Li, R. Yu, H. Lei, and W. Yu, Phys. Rev. Lett. 117, 237001 (2016).

[11] J. W. Lynn and P. Dai, Physica C (Amsterdam) 469, 469 (2009).

[12] W. Bao, Y. Qiu, Q. Huang, M. A. Green, P. Zajdel, M. R. Fitzsimmons, M. Zhernenkov, S. Chang, M. Fang, B. Qian, E. K. Vehstedt, J. Yang, H. M. Pham, L. Spinu, and Z. Q. Mao, Phys. Rev. Lett. 102, 247001 (2009).

[13] S. Li, C. de la Cruz, Q. Huang, Y. Chen, J. W. Lynn, J. Hu, Y.-L. Huang, F.-C. Hsu, K.-W. Yeh, M.-K. Wu, and P. Dai, Phys. Rev. B 79, 054503 (2009).

[14] F. Ma, W. Ji, J. Hu, Z.-Y. Lu, and T. Xiang, Phys. Rev. Lett. 102, 177003 (2009).

[15] H.-Y. Cao, S. Chen, H. Xiang, and X.-G. Gong, Phys. Rev. B 91, 020504(R) (2015).

[16] K. Liu, M. Gao, Z.-Y. Lu, and T. Xiang, Chin. Phys. B 24, 117402 (2015).

[17] C. Tresca, F. Ricci, and G. Profeta, 2D Materials 2, 015001 (2015).

[18] S. Wang and F. Wang, arXiv:1510.05476.

[19] M. H. Christensen, J. Kang, B. M. Andersen, I. Eremin, and R. M. Fernandes, Phys. Rev. B 92, 214509 (2015).

[20] M. Ma, D. Yuan, Y. Wu, H. Zhou, X. Dong, and F. Zhou, Supercond. Sci. Technol. 27, 122001 (2014).

[21] See Supplemental Material at http://link.aps.org/supplemental/ 10.1103/PhysRevB.95.180504 for the sample preparation pro- cedure, description of the $\mu \mathrm{SR}$ under pressure technique and calculations of $f_{\text {osc }}(\Theta)$ for 101 oriented sample.

[22] R. Khasanov, Z. Guguchia, A. Maisuradze, D. Andreica, M. Elender, A. Raselli, Z. Shermadini, T. Goko, E. Morenzoni, and A. Amato, High Press. Res. 36, 140 (2016).

[23] R. Khasanov, M. Bendele, K. Conder, H. Keller, E. Pomjakushina, and V. Pomjakushin, New J. Phys. 12, 073024 (2010).

[24] K. Momma and F. Izumi, J. Appl. Crystallogr. 41, 653 (2008).

[25] S. J. Blundell, Physica B (Amsterdam) 404, 581 (2009).

[26] A. Maisuradze, Z. Guguchia, B. Graneli, H. M. Ronnow, H. Berger, and H. Keller, Phys. Rev. B 84, 064433 (2011).

[27] A. Amato, P. Dalmas de Réotier, D. Andreica, A. Yaouanc, A. Suter, G. Lapertot, I. M. Pop, E. Morenzoni, P. Bonfà, F. Bernardini, and R. De Renzi, Phys. Rev. B 89, 184425 (2014).

[28] R. Khasanov, A. Amato, P. Bonfà, Z. Guguchia, H. Luetkens, E. Morenzoni, R. De Renzi, and N. D. Zhigadlo, Phys. Rev. B 93, 180509(R) (2016).

[29] Q. Wang, Y. Shen, B. Pan, Y. Hao, M. Ma, F. Zhou, P. Steffens, K. Schmalzl, T. R. Forrest, M. Abdel-Hafiez, X. Chen, D. A. Chareev, A. N. Vasiliev, P. Bourges, Y. Sidis, H. Cao, and J. Zhao, Nat. Mater. 15, 159 (2016).

[30] M. Ma, P. Bourges, Y. Sidis, Y. Xu, S. Li, B. Hu, J. Li, F. Wang, and Y. Li, arXiv:1610.01277.

[31] J. K. Glasbrenner, I. I. Mazin, H. O. Jeschke, P. J. Hirschfeld, R. M. Fernandes, and Roser Valentí, Nat. Phys. 11, 953 (2014).

[32] T. Terashima, N. Kikugawa, A. Kiswandhi, D. Graf, E.-S. Choi, J. S. Brooks, S. Kasahara, T. Watashige, Y. Matsuda, T. Shibauchi, T. Wolf, A. E. Böhmer, F. Hardy, C. Meingast, H. V. Löhneysen, and S. Uji, Phys. Rev. B 93, 094505 (2016). 